\title{
Second-Order Super-Twisting Sliding Mode Control for Finite-Time Leader-Follower Consensus with Uncertain Nonlinear Multiagent Systems
}

\author{
Nan Liu, ${ }^{1,2}$ Rui Ling, ${ }^{1,2}$ Qin Huang, ${ }^{1}$ and Zheren Zhu' \\ ${ }^{1}$ College of Automation, Chongqing University, Chongqing 400044, China \\ ${ }^{2}$ Key Laboratory for Spacecraft TT\&C and Communication under the Ministry of Education, Chongqing 400044, China \\ Correspondence should be addressed to Rui Ling; rui.ling@cqu.edu.cn
}

Received 18 September 2014; Revised 13 December 2014; Accepted 29 December 2014

Academic Editor: Peng Shi

Copyright (C) 2015 Nan Liu et al. This is an open access article distributed under the Creative Commons Attribution License, which permits unrestricted use, distribution, and reproduction in any medium, provided the original work is properly cited.

Consensus tracking problem of the leader-follower multiagent systems is resolved via second-order super-twisting sliding mode control approach. The followers' states can keep consistent with the leader's states on sliding surfaces. The proposed approach can ensure the finite-time consensus if the directed graph of the nonlinear system has a directed path under the condition that leader's control input is unavailable to any followers. It is proved by using the finite-time Lyapunov stability theory. Simulation results verify availability of the proposed approach.

\section{Introduction}

Recently, cooperative control of multiagent systems (MAS) received a lot of interest, such as consensus, containment control, formation control, coverage control, and flocking [1, 2]. It has attracted a lot of researchers due to having potential application in many fields. Compared with traditional control systems, agents in MAS need to work together cooperatively and achieve a common goal with shared information, such as position, speed, or other parameters, in spite of limited and unreliable communication. There has been many different control strategies, such as graph theory approach [3-5], decentralized control approach [6, 7], and virtual leader approach [8]. The application of this research involves unmanned air vehicles [9], cooperative robotic systems [4, 8], and so forth. Such control strategies have many advantages, such as easy implementation and low cost [10]. MAS based on positive systems was investigated [11]. Various results from formation control of MAS are addressed as well.

The consensus tracking is an interesting problem in leader-follower MAS. Agents in MAS agree on a common value with cooperative control law or consensus protocol. A summary of approaches for consensus algorithms was introduced [12]. The consensus tracking algorithm was proposed and analyzed under variable undirected network topologies [13-15]. Some control approaches for MAS were presented $[16,17]$, provided that necessary and sufficiency conditions were fulfilled, to achieve consensus under directed communication topologies. A sliding mode control (SMC) with multisurface approach for leader-follower MAS was proposed to achieve the convergence in finite time [18]. Tracking errors of first-order or second-order agents in MAS can be forced to zero under directed fixed and switching network topologies.

It is necessary for consensus algorithm to achieve the convergence in finite time even if there are external disturbances and system uncertainties in many applications. Compared with the infinite time property, finite-time leader-follower consensus can perform better, such as faster convergence rate. This paper aims to research finite-time consensus for MAS.

As is well known, traditional sliding mode control (SMC) $[19,20]$ was robust against parameter uncertainty and external disturbances. It has been applied in many fields, such as aircrafts, electrical motors, and power systems [21]. But it is not common in the field of multiagent networks. And traditional SMC may cause high-frequency chattering in the 
vicinity of the sliding surfaces [22]. The chattering problem is main drawback for traditional SMC. Some approaches were proposed to attenuate chattering phenomenon, such as high-order sliding mode control (HOSMC). It features a continuous signal instead of switching signal [23]. This approach not only can maintain the merits of the traditional method but also can attenuate chattering. Second-order sliding mode control (SOSMC) is a class of HOSMC, such as super-twisting algorithm [24], suboptimal algorithm [25], improved suboptimal algorithm [25], and twisting algorithm [26]. Twisting algorithm needs the sign of $\dot{s}$. Suboptimal algorithm has memory characteristics through the most recent singular point. Super-twisting algorithm takes advantage of the fact that it steers the sliding variable to zero for the systems with relative degree of two without the time derivative of sliding variable.

This paper adapts second-order super-twisting SMC to achieve the consensus tracking for leader-follower MAS in finite time and gives the condition of leader-follower consensus.

This paper is organized as follows. Several concepts and theories are presented in Section 2. Section 3 is the problem statement. In Section 4, the proposed approach for leaderfollower consensus based on second-order super-twisting algorithm was presented, convergence analysis of the MAS was provided, and the condition of the finite-time consensus for leader-follower MAS is derived. Subsequently simulation results are presented in Section 5. Section 6 gives conclusion.

\section{Preliminaries}

This section gives several preliminary concepts and theories in order to facilitate the subsequent analysis.

2.1. Concepts in Graph Theory for MAS. A directed graph $\mathscr{G}=(\mathscr{V}, \mathcal{\varepsilon}, \mathscr{A})$ can be adapted to express the communication between the agents, where $\mathscr{V}=\{0,1,2, \ldots, m\}$ is the set of nodes and $\varepsilon$ is the set of edges. The edge in directed graph $\mathscr{G}$ is denoted by the sequence of edges $\left(h_{1}, h_{2}\right),\left(h_{2}, h_{3}\right), \ldots,\left(h_{m-1}, h_{m}\right)$ if and only if the agents can exchange information with each other. $\mathscr{A}=\left[a_{h j}\right]$ is called the weighted adjacency matrix, if $\varepsilon_{h j}=(h, j) \in \varepsilon$, and there exists edge between node $j$ and node $h$; then $a_{h j}>0$, and $a_{h j}=0$ otherwise:

$$
\mathscr{A}=\left[\begin{array}{cccc}
0 & 0 & \cdots & 0 \\
a_{10} & a_{11} & \cdots & a_{1 m} \\
\vdots & \ddots & \ddots & \vdots \\
a_{m 0} & a_{m 1} & \cdots & a_{m m}
\end{array}\right] \in \mathfrak{R}^{(m+1) \times(m+1)}
$$

Define diagonal matrix $\mathscr{D}=\operatorname{diag}\left\{d_{0}, d_{1}, \ldots, d_{m}\right\}$, and $d_{h}=$ $\sum_{j=0}^{m} a_{h j}$ for $h=0,1, \ldots, m$. Define directed graph $\mathscr{G}$ 's Laplacian matrix:

$$
\mathscr{L}=\mathscr{D}-\mathscr{A} \in \mathfrak{R}^{(m+1) \times(m+1)}
$$

Define $b_{h}$ that is a connection weight between agent $h$ and leader. $b_{h}>0$, if there exists an edge between the agent $h$ and leader; otherwise $b_{h}=0$ [27]. Let

$$
\overline{\mathscr{B}}=\operatorname{diag}\left\{b_{1}, b_{2}, \ldots, b_{m}\right\} \text {. }
$$

Define $\overline{\mathscr{G}}=\{\overline{\mathscr{V}}, \bar{\varepsilon}, \overline{\mathscr{A}}\}$ that is the subgraph of $\mathscr{G}$, consisting of $m$ followers, where

$$
\overline{\mathscr{A}}=\left[\begin{array}{ccc}
a_{11} & \cdots & a_{1 m} \\
\vdots & \ddots & \vdots \\
a_{m 1} & \cdots & a_{m m}
\end{array}\right] \in \mathfrak{R}^{m \times m}
$$

Moreover, let $\overline{\mathscr{D}}=\operatorname{diag}\left\{\bar{d}_{1}, \bar{d}_{2}, \ldots, \bar{d}_{m}\right\}$, and $\bar{d}_{h}=\sum_{j=1}^{m} a_{h j}$ for $h=1,2, \ldots, m$. Defining the Laplacian of subgraph $\overline{\mathscr{G}}$,

$$
\overline{\mathscr{L}}=\overline{\mathscr{D}}-\overline{\mathscr{A}} \in \mathfrak{R}^{m \times m}
$$

Theorem 1. If graph $\mathscr{G}=(\mathscr{V}, \varepsilon, \mathscr{A})$ has a directed path, then $[\overline{\mathscr{L}}+\overline{\mathscr{B}}]$ is a nonsingular matrix $[18,28]$.

2.2. Finite-Time Lyapunov Stability Theory. Consider a nonlinear system which satisfies

$$
\dot{x}=f(x) .
$$

Assume that $f(x)$ is non-Lipschitz continuous with respect to $x$. A point $x^{*} \in R^{n}$ is an equilibrium point of (6) if $f\left(x^{*}\right) \equiv$ 0 . The point $x^{*}$ is locally asymptotically stable, while $t \rightarrow$ $\infty$. Finite-time stability requires all solution trajectories of (6) trend toward the origin in finite time $[29,30]$. The sufficient conditions are as follows.

Theorem 2 (see [27]). Consider the nonlinear system (6). Choose a Lyapunov function $V(x)$, real number $\alpha>0$, and $0<\theta<1$, so that

$$
\begin{aligned}
& \text { (1) } V(x) \text { is positive, } \\
& \text { (2) } \dot{V}(x) \leq-\alpha V^{\theta}(x) \text {. }
\end{aligned}
$$

Then, it is finite-time stable at the origin. The settling time related to $x(0)=x^{*}$ satisfies

$$
T\left(x^{*}\right) \leq \frac{V^{1-\theta}\left(x^{*}\right)}{\alpha(1-\theta)} .
$$

\section{Problem Statement}

The state of $m$ followers can keep changing and track a leader by communicating with each other in leader-follower MAS. The topology of agents can be denoted by a graph $\mathscr{G}$. The leader's behavior is not affected by the followers' behavior. However, the latter's behavior is influenced by that of the leader. The target of consensus tracking is that a leader moves independently of other followers and the control strategy must guarantee the followers' states to be consistent with leader's states. This characteristic is useful to regulate all agents in MAS $[31,32]$ because the control objective of the 
agents is able to be achieved easily by only regulating the leader. Finite-time consensus tracking requires the followers to finish this transient in finite time.

The task is to design a control law to guarantee finite-time consensus for leader-follower MAS. The leader's dynamic in MAS is expressed by the uncertain nonlinear system:

$$
\begin{gathered}
\dot{x}^{0}=v^{0}, \\
\dot{v}^{0}=g^{0}\left(x^{0}, t\right) u^{0},
\end{gathered}
$$

where $x^{0}, u^{0}$ are the leader's state and control input, respectively, and $g^{0}\left(x^{0}, t\right)$ is an uncertain function.

The dynamic of the followers in MAS [33] is described by the following uncertain nonlinear system:

$$
\begin{gathered}
\dot{x}^{h}=v^{h}, \\
\dot{v}^{h}=g^{h}\left(x^{h}, t\right) u^{h}+d^{h}\left(x^{h}, t\right),
\end{gathered}
$$

where the position information of agent $h$ is denoted by $X=\left[x^{1}, x^{2}, \ldots, x^{h}\right]^{T}, v^{h} \in R$ is the velocity of the agents, $u^{h}$ represent the control input, $g^{h}\left(x^{h}, t\right)$ and $d^{h}\left(x^{h}, t\right)$ are uncertain function, and $d^{h}\left(x^{h}, t\right)$ indicates the external disturbance.

The control input $u^{h}$ is only determined by the relative information between itself and its neighbors, since $u^{0}$ is not available by the followers even though they may be related to the leader. The control objective is to present a second-order super-twisting SMC algorithm for a given target system (9) so that the agents' position and velocity satisfy the following equation: $\lim _{t \rightarrow T}\left|x^{h}(t)-x^{0}(t)\right|=0, \lim _{t \rightarrow T}\left|v^{h}(t)-v^{0}(t)\right|=0$. Then the followers' position and velocity will keep consistent with those of the leader in finite time.

\section{Controller Design}

4.1. Control Objective. Traditional SMC takes advantage of the fact that it is robust against uncertainties and external disturbances, assuming that system's uncertainties and disturbances are bounded. Due to the discontinuousness of control actions, the chattering problem will occur when the trajectory of system moves in the vicinity of the sliding surface.

SOSMC can maintain the merits of traditional SMC, and it can attenuate chattering phenomenon as well. Moreover, traditional SMC needs its $(r-1)$ order sliding variables for system with relative degree $r(r>1)$. However, supertwisting algorithm only requires its $(r-2)$ order sliding variables for the same system. In the actual engineering application, it can save the cost with this algorithm due to reduction in the number of sensors. In this paper, a smooth implementable robust control is presented to achieve leaderfollower consensus in finite time.

4.2. Super-Twisting Algorithm. Consider the uncertain nonlinear system (9); also it can be described as $\dot{x}^{h}=f\left(t, x^{h}, u^{h}\right)$. The objective of SMC is achieved by making the state trajectory on sliding surfaces in the state plane. Then sliding surfaces can be defined as

$$
\begin{gathered}
S=\left[s_{1}, s_{2}, \ldots, s_{n}\right]^{T}, \\
s_{i}=e_{i}{ }^{\mathrm{Q}}+c \dot{e}_{i} \quad i=1, \ldots, n,
\end{gathered}
$$

where $c$ is a positive constant and $0<Q<1$. Define the error function as

$$
e_{i}=\sum_{j=1}^{m} a_{h j}\left(x^{h}-x^{j}\right)+b_{h}\left(x^{h}-x^{0}\right) .
$$

By differentiating $s_{i}$ twice, the following equations can be obtained:

$$
\begin{aligned}
\dot{s}_{i}= & \dot{s}_{i}\left(x^{h}(t), t, u^{h}(t)\right) \\
= & \frac{\partial}{\partial t} s_{i}\left(x^{h}, t\right)+\frac{\partial}{\partial x^{h}} s_{i}\left(x^{h}, t\right) f\left(x^{h}, t, u^{h}\right), \\
\ddot{s}_{i}= & \ddot{s}_{i}\left(x^{h}(t), t, \dot{u}^{h}(t)\right) \\
= & \frac{\partial}{\partial t} \dot{s}_{i}\left(x^{h}, t, u^{h}\right)+\frac{\partial}{\partial x^{h}} \dot{s}_{i}\left(x^{h}, t, u^{h}\right) f\left(x^{h}, t, u^{h}\right) \\
& +\frac{\partial}{\partial u^{h}} \dot{s}_{i}\left(x^{h}, t, u^{h}\right) \dot{u}^{h}(t) .
\end{aligned}
$$

Assume that the following conditions hold [34].

(1) In the set $U=\left\{u^{h}(t):\left|u^{h}(t)\right| \leq U_{M}\right\}, U_{M}>1$ is a constant; $u^{h}(t)$ is continuous for all $t$, while for all $t u^{h}(t) \in U$.

(2) There exists $u_{1}^{h} \in(0,1)$, and $u^{h}(t)$ with $\left|u^{h}(t)\right|>u_{1}^{h}$, $s_{i}(t) u^{h}(t)>0$ for $t>t_{1}$. Therefore, the control input $u^{h}(t)=-\operatorname{sign}\left(s_{i}\left(t_{0}\right)\right)$, in which $t_{0}$ is the initial time, provided that the sliding surface $s_{i}=0$ in finite time.

(3) exist positive constants $s_{0}, u^{0}<1, \Gamma_{m}$, and $\Gamma_{M}$; if $\left|s_{i}\left(x^{h}, t\right)\right|<s_{0}$, then

$$
0<\Gamma_{m}<\frac{\partial}{\partial u^{h}} \dot{s}_{i}\left(x^{h}, t, u^{h}\right)<\Gamma_{M}, \quad \forall u^{h} \in U, x^{h} \in X
$$

and the inequality $\left|u^{h}(t)\right|>u^{0}$ requires $\dot{s}_{i} u^{h}>0$.

(4) There are $\left|s_{i}\left(x^{h}, t\right)\right|<s_{0}$ and a positive real number $\Phi$, and the following inequality satisfies, for all $t, x^{h} \in X$, $u^{h} \in U$ :

$$
\left|\frac{\partial}{\partial t} \dot{s}_{i}\left(x^{h}, t, u^{h}\right)+\frac{\partial}{\partial x^{h}} \dot{s}_{i}\left(x^{h}, t, u^{h}\right) f\left(x^{h}, t, u^{h}\right)\right| \leq \Phi .
$$

Consider the sliding variables $s_{i}$ and $\dot{s}_{i}$, and the system is described as follows:

$$
\ddot{s}_{i}=\varphi\left(t, x^{h}\right)+\gamma\left(t, x^{h}\right) \dot{u}^{h},
$$

where $\dot{s}_{i}$ is immeasurable, but the sign is known, and $\varphi\left(t, x^{h}\right)$ and $\gamma\left(t, x^{h}\right)$ are uncertain functions with

$$
\Phi>0, \quad\left|\varphi\left(t, x^{h}\right)\right| \leq \Phi, \quad 0<\Gamma_{m}<\gamma\left(t, x^{h}\right)<\Gamma_{M} .
$$




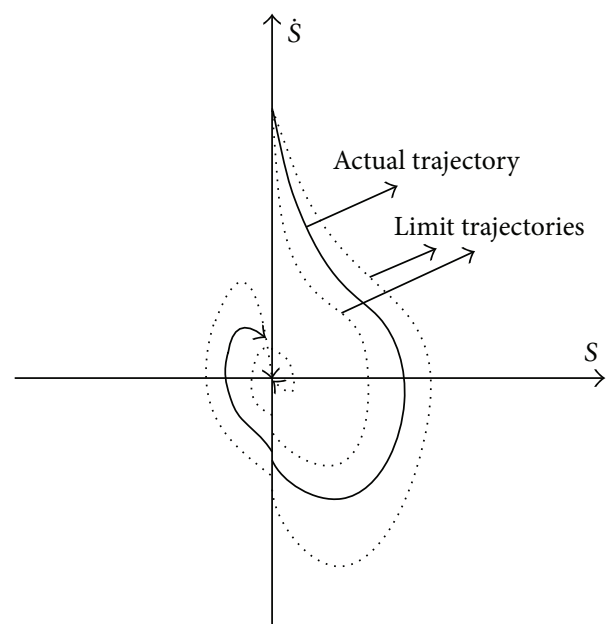

FIGURE 1: Super-twisting algorithm typical trajectory on phase plane.

The super-twisting control $u^{h}$ can be described as [34]:

$$
\begin{aligned}
& u^{h}=u_{1}+u_{2}, \\
& \dot{u}_{1}=-W \operatorname{sign}\left(s_{i}\right), \\
& u_{2}=-\lambda\left|s_{i}\right|^{\rho} \operatorname{sign}\left(s_{i}\right),
\end{aligned}
$$

where $\lambda>0, W>0$.

The trajectory can converge to the sliding surface under the following conditions:

$$
\begin{gathered}
W>\frac{\Phi}{\Gamma_{m}} \\
\lambda^{2} \geq \frac{4 \Phi}{\Gamma_{m}^{2}} \frac{\Gamma_{M}(W+\Phi)}{\Gamma_{m}(W-\Phi)}, \\
0<\rho \leq 0.5
\end{gathered}
$$

The control law (17) includes two parts. The first part is a function of discontinuous derivative of sliding variable and the second is described by a continuous function of sliding variable. The typical trajectories on phase plane are depicted in Figure 1, which twist around the origin.

According to conditions (3) and (4), we can obtain that the solid line represents actual trajectory and the dotted line represents limit trajectories in Figure 1.

Theorem 3. According to the dynamic of agents (8) and (9) with external disturbance $d^{h}$, if there is a directed graph $\mathscr{G}$ with directed paths, then

$$
\lim _{t \rightarrow T}\left|x^{h}(t)-x^{0}(t)\right|=0, \quad \lim _{t \rightarrow T}\left|v^{h}(t)-v^{0}(t)\right|=0 .
$$

That is, it implies that differences between leader's and followers' states converge toward zero in finite time.
Proof. Take

$$
\begin{aligned}
& \varepsilon_{1}=\left[x^{1}, x^{2}, \ldots, x^{h}\right]^{T}-x^{0} 1, \\
& \varepsilon_{2}=\left[v^{1}, v^{2}, \ldots, v^{h}\right]^{T}-v^{0} 1
\end{aligned}
$$

The error dynamic of the agent can be expressed as

$$
\begin{aligned}
& \dot{\varepsilon}_{1}=\varepsilon_{2}, \\
& \dot{\varepsilon}_{2}=g^{h} u^{h}+d^{h}-u^{0} 1 .
\end{aligned}
$$

And the second-order super-twisting sliding variable of system (21) is modified to

$$
S=(\overline{\mathscr{L}}+\overline{\mathscr{B}}) \varepsilon_{1}^{\mathrm{Q}}+(\overline{\mathscr{L}}+\overline{\mathscr{B}}) c \varepsilon_{2},
$$

where sliding variable $S=\left[s_{1}, s_{2}, \ldots, s_{n}\right]^{T}$.

The sliding surfaces can be defined as $S=0$ or

$$
S=(\overline{\mathscr{L}}+\overline{\mathscr{B}}) \varepsilon_{1}^{Q}+(\overline{\mathscr{L}}+\overline{\mathscr{B}}) c \varepsilon_{2}=0 .
$$

According to Theorem 1 , we can get that the matrix $(\overline{\mathscr{L}}+\overline{\mathscr{B}})$ is invertible on the sliding surfaces. Due to expression (23), the error vector $\varepsilon_{2}$ can be modified to

$$
\varepsilon_{2}=-\frac{1}{c} \varepsilon_{1}^{Q}
$$

Owing to (24), it is easy to see that $\varepsilon_{2}$ is the linear relationship with $\varepsilon_{1}$. Therefore, in order to prove the leader-follower consensus of MAS upon sliding surfaces in finite time, $\varepsilon_{1}$ should converge to zero in finite time.

Choosing a Lyapunov function,

$$
V=\frac{1}{2} \varepsilon_{1}^{T} \varepsilon_{1} .
$$

We can get

$$
\begin{aligned}
\dot{V} & =\varepsilon_{1}^{T} \dot{\varepsilon}_{1}=\varepsilon_{1}^{T} \varepsilon_{2} \\
& =-\frac{1}{c} \varepsilon_{1}^{T} \varepsilon_{1}^{Q}=-\frac{1}{c}(2 V)^{(1+Q) / 2}<0 .
\end{aligned}
$$

According to expression (26), we can get

$$
\dot{V}+\frac{1}{c}(2 V)^{(1+Q) / 2}=0 .
$$

Hence, according to Theorem 2 , we can get $\varepsilon_{1} \rightarrow 0$ in finite time:

$$
T=\frac{c \cdot(2 V)^{(1-Q) / 2}\left(x^{*}\right)}{1-Q} .
$$

Thus, in finite time $T, \lim _{t \rightarrow T}\left|x^{h}(t)-x^{0}(t)\right|=0$, $\lim _{t \rightarrow T}\left|v^{h}(t)-v^{0}(t)\right|=0$, which completes the proof of Theorem 3, and the states of followers keep up with that of leader after a finite time $T$. It implies that the followers' states can keep consistent with the leader's states in finite time. 


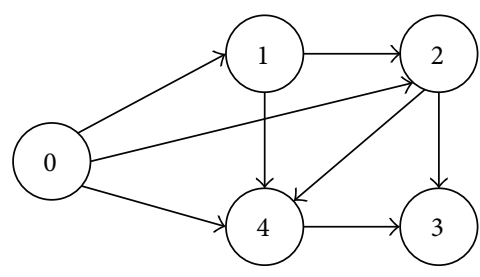

FIGURE 2: Directed fixed graph.

\section{Simulation}

The leader's dynamics indexed by 0 are as follows:

$$
\begin{aligned}
& \dot{x}^{0}=v^{0}, \\
& \dot{v}^{0}=u^{0}\left(\sin \left(x^{0}\right)\right)^{1 / 2},
\end{aligned}
$$

where $u^{0}$ is chosen as

$$
u^{0}=-\frac{\sin \left(x^{0}\right)}{1+e^{-t}} .
$$

The uncertain nonlinear dynamics of follower $h$ can be given by

$$
\begin{aligned}
& \dot{x}^{h}=v^{h}, \\
& \dot{v}^{h}=u^{h}\left(\sin \left(x^{h}\right)\right)^{1 / 2}+d^{h},
\end{aligned}
$$

where the external disturbance $d^{h}$ can be assumed to be bounded by $\left|d^{h}\right| \leq 1$, where $d^{h}=\sin \left(\cos \left(t+x^{h}\right)\right)$ is chosen. Consider the initial values of agents indexed by $0,1,2,3$, and 4 [18]:

$$
x^{0}=\frac{\pi}{2}, \quad x^{1}=1, \quad x^{2}=2, \quad x^{3}=3, \quad x^{4}=-1 .
$$

We consider a directed fixed graph to represent the exchange of information among the agents in Figure 2, and the state of leader is only available to followers 1,2 , and 4 .

In order to demonstrate the proposed control algorithm's capability in leader-follower MAS under switching topologies, we suppose that the directed switching graphs from the set $\mathscr{G}=\left(\mathscr{G}_{1}, \mathscr{G}_{2}, \mathscr{G}_{3}, \mathscr{G}_{4}, \mathscr{G}_{5}, \mathscr{G}_{6}\right)$ are given by Figure 3 , in which the switching time intervals are $[0,3),[3,5),[5,7),[7,9),[9,12),[12,15]$. And the controller parameters are listed in Table 1.

The simulation results demonstrate finite-time convergence of the leader-follower MAS. Figures 4 and 6 illustrate the trajectories of states and sliding variables with fixed topology and switching topologies. The position information between four followers and leader is illustrated, respectively, in Figures 4(a) and 6(a). Four followers finally catch up with the leader. It can be seen that the followers with external disturbances can accurately catch up with the leader under a directed fixed topology and switching topologies. Figures 4(b) and 6(b) show that the sliding variables ultimately
TABLE 1: Controller parameters.

\begin{tabular}{lc}
\hline Parameter & Value \\
\hline$W$ & 3 \\
$\lambda$ & 15 \\
$\rho$ & 15 \\
$c$ & 0.5 \\
\hline
\end{tabular}

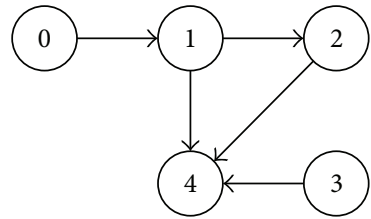

(a) $\mathscr{G}_{1}$

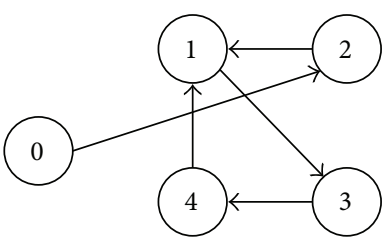

(c) $\mathscr{G}_{3}$

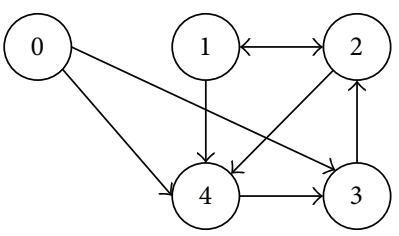

(e) $\mathscr{G}_{5}$

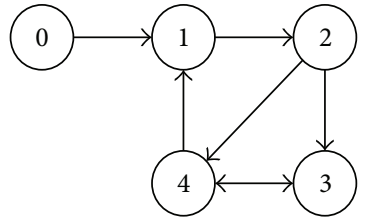

(b) $\mathscr{G}_{2}$

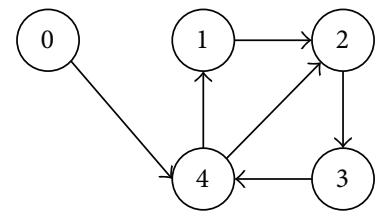

(d) $\mathscr{G}_{4}$

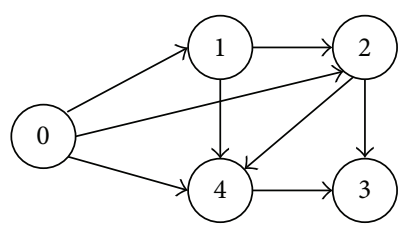

(f) $\mathscr{G}_{6}$
FIGURE 3: Directed switching graph.

converge to zero on the sliding surfaces. Also, the trajectories of sliding variables on $s$-s state plane are illustrated in Figures 5 and 7, representing directed fixed graph and switching graphs, respectively. All of sliding variables converge to zero eventually.

\section{Conclusions}

Consensus tracking control of MAS has been investigated in this paper. Second-order super-twisting algorithm is adapted for the leader-follower MAS to make each agent track the leader with nonzero input in finite time under directed fixed topology and switching topologies. Moreover, it has been proved that, under sufficient conditions, second-order super-twisting SMC approach allows the agents' position and velocity errors to zero with external disturbance in finite time. Simulation verified the availability of second-order supertwisting SMC algorithm.

\section{Conflict of Interests}

The authors declare that there is no conflict of interests regarding the publication of this paper. 


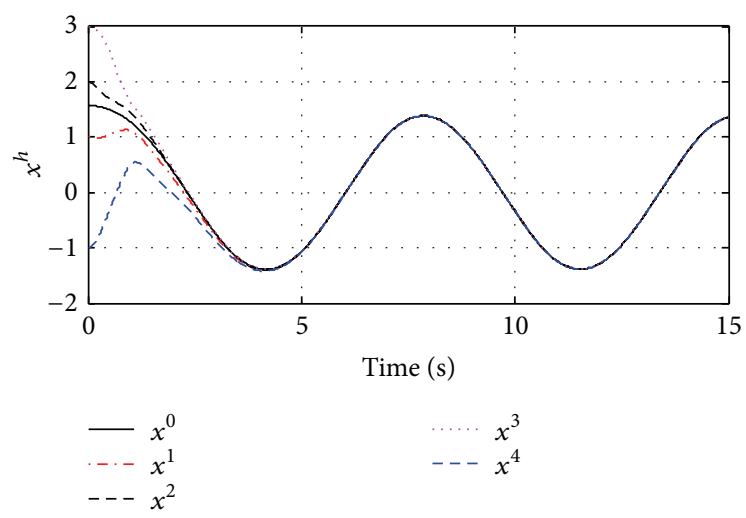

(a) Performance of $x^{h}$
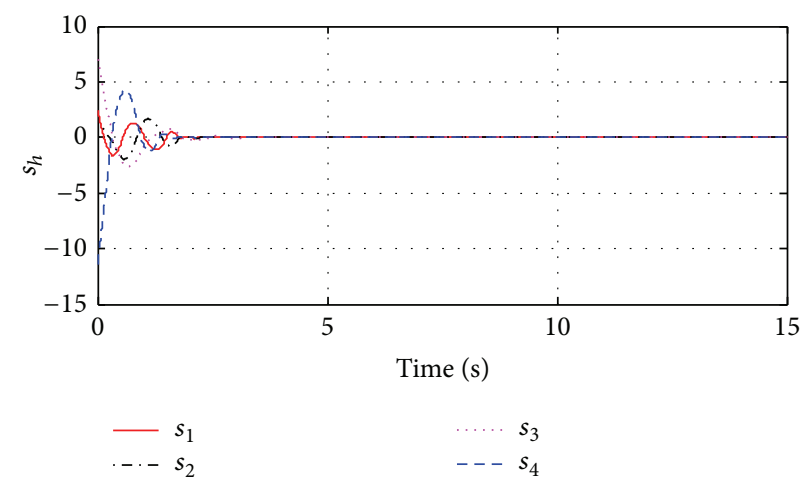

(b) Sliding surfaces

FIGURE 4: Finite-time leader-follower consensus with external disturbances by fixed topology.

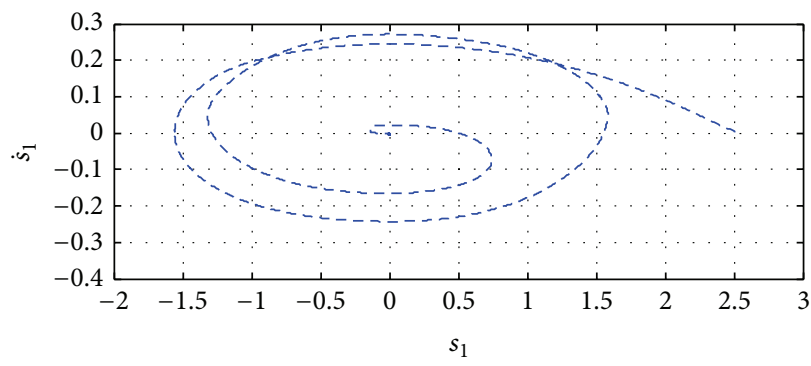

(a) Performance of $s_{1}, \dot{s}_{1}$

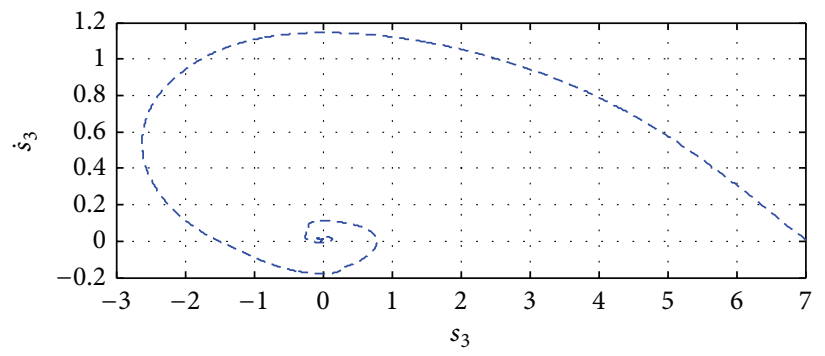

(c) Performance of $s_{3}, \dot{s}_{3}$

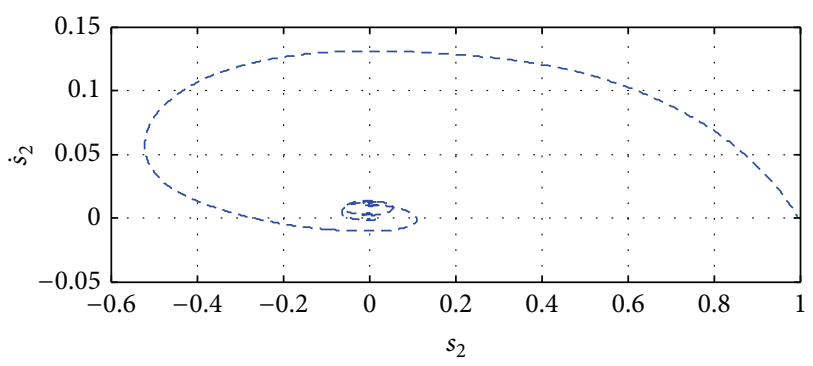

(b) Performance of $s_{2}, \dot{s}_{2}$

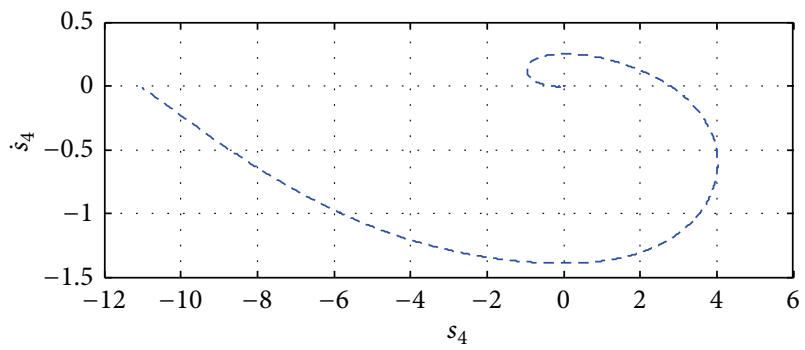

(d) Performance of $s_{4}, \dot{s}_{4}$

FIGURE 5: Super-twisting algorithm $s$-s state plane trajectories with fixed topology.

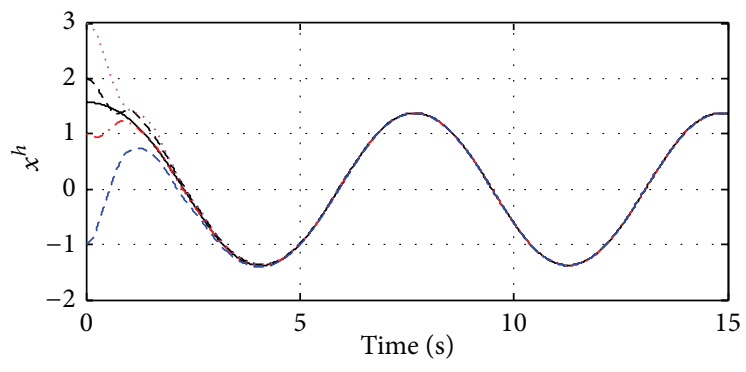

$\begin{array}{ll}- & x^{0} \\ -\cdot-x^{1} & ---x^{4} \\ ---x^{2} & \end{array}$

(a) Performance of $x^{h}$
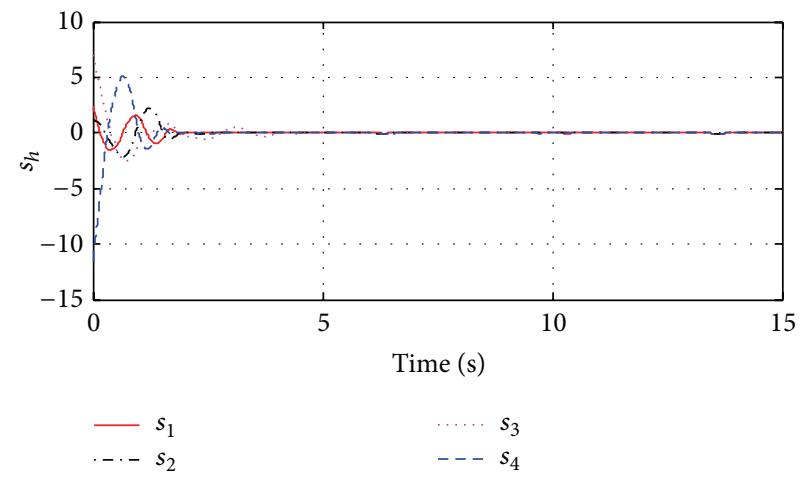

(b) Sliding surfaces

FIGURE 6: Finite-time leader-follower consensus with external disturbances by directed switching topology. 


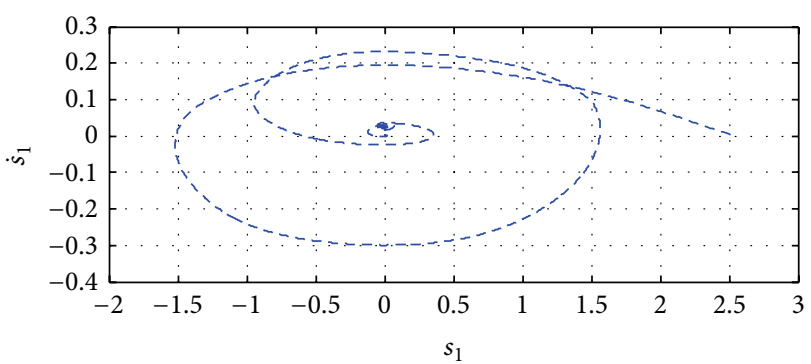

(a) Performance of $s_{1}-\dot{s}_{1}$

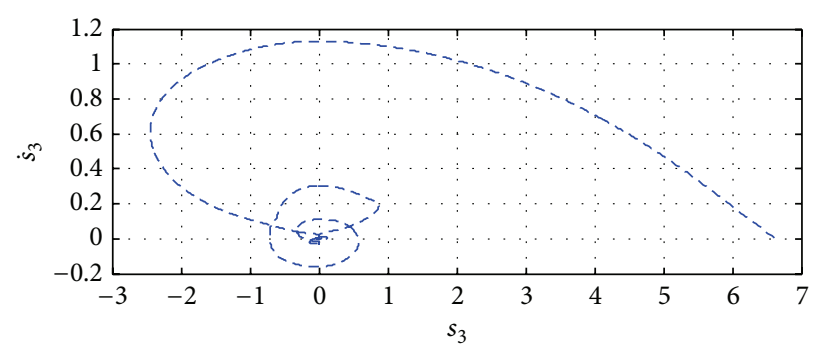

(c) Performance of $s_{3}-\dot{s}_{3}$

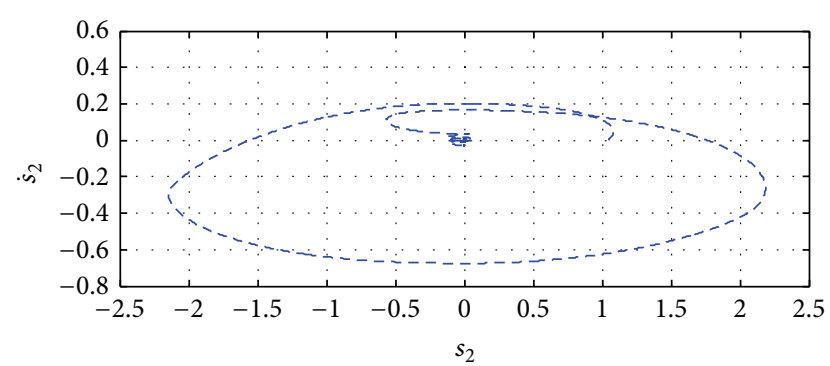

(b) Performance of $s_{2}-\dot{s}_{2}$

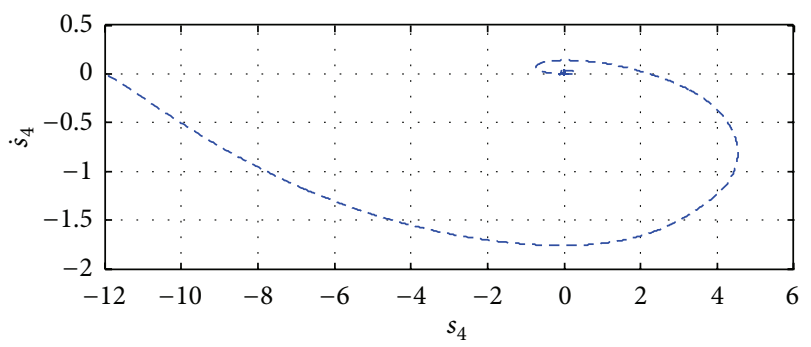

(d) Performance of $s_{4}-\dot{s}_{4}$

FIGURE 7: Super-twisting algorithm $s-\dot{s}$ state plane trajectories with directed switching topologies.

\section{Acknowledgments}

This work was supported in part by National Natural Science Foundation of China (61203097), China Postdoctoral Science Foundation (2013T60840 and 2012M511907), Fundamental Research Funds for the Central Universities in China (106112013CDJZR170003), AQSIQ Science and Technology Projects (2012IK029), Open Research Fund Program of Key Lab. for Spacecraft TT\&C and Communication, Ministry of Education, China (CTTC-FX201304), and Chongqing Application Development Plan Project in China (CSTC2014YYKFA80017).

\section{References}

[1] R. Olfati-Saber, "Flocking for multi-agent dynamic systems: algorithms and theory," IEEE Transactions on Automatic Control, vol. 51, no. 3, pp. 401-420, 2006.

[2] G. Shi and Y. Hong, "Global target aggregation and state agreement of nonlinear multi-agent systems with switching topologies," Automatica, vol. 45, no. 5, pp. 1165-1175, 2009.

[3] L. Cheng, Z.-G. Hou, and M. Tan, "Decentralized adaptive leader-follower control of multi-manipulator system with uncertain dynamics," in Proceedings of the 34th Annual Conference of the IEEE Industrial Electronics Society (IECON '08), pp. 1608-1613, Orlando, Fla, USA, November 2008.

[4] Z.-G. Hou, L. Cheng, and M. Tan, "Multicriteria optimization for coordination of redundant robots using a dual neural network," IEEE Transactions on Systems, Man, and Cybernetics, Part B: Cybernetics, vol. 40, no. 4, pp. 1075-1087, 2010.

[5] L. Cheng, Z.-G. Hou, Y. Lin, M. Tan, and W. J. Zhang, "Solving a modified consensus problem of linear multi-agent systems," Automatica, vol. 47, no. 10, pp. 2218-2223, 2011.

[6] Z.-G. Hou, L. Cheng, and M. Tan, "Decentralized robust adaptive control for the multiagent system consensus problem using neural networks," IEEE Transactions on Systems, Man, and Cybernetics, Part B: Cybernetics, vol. 39, no. 3, pp. 636-647, 2009.

[7] L. Cheng, Z.-G. Hou, M. Tan, Y. Lin, and W. J. Zhang, "Neural-network-based adaptive leader-following control for multiagent systems with uncertainties," IEEE Transactions on Neural Networks, vol. 21, no. 8, pp. 1351-1358, 2010.

[8] L. Cheng, Z.-G. Hou, and M. Tan, "Decentralized adaptive consensus control for multi-manipulator system with uncertain dynamics," in Proceedings of the IEEE International Conference on Systems, Man and Cybernetics (SMC '08), pp. 2712-2717, Singapore, October 2008.

[9] M. E. Campbell and W. W. Whitacre, "Cooperative tracking using vision measurements on SeaScan UAVs," IEEE Transactions on Control Systems Technology, vol. 15, no. 4, pp. 613-626, 2007.

[10] N. E. Leonard, D. A. Paley, F. Lekien, R. Sepulchre, D. M. Fratantoni, and R. E. Davis, "Collective motion, sensor networks, and ocean sampling," Proceedings of the IEEE, vol. 95, no. 1, pp. 4874, 2007.

[11] Y. Ebihara, D. Peaucelle, and D. Arzelier, "Stability and persistence analysis of large scale interconnected positive systems," in Proceedings of the 12th European Control Conference (ECC '13), pp. 3366-3371, July 2013.

[12] R. Olfati-Saber, J. A. Fax, and R. M. Murray, "Consensus and cooperation in networked multi-agent systems," Proceedings of the IEEE, vol. 95, no. 1, pp. 215-233, 2007.

[13] Y. Cao and W. Ren, "Distributed coordinated tracking via a variable structure approach-part I: consensus tracking," in Proceedings of the American Control Conference (ACC '10), pp. 4744-4749, Baltimore, Md, USA, June 2010.

[14] Y. Cao and W. Ren, "Distributed coordinated tracking via a variable structure approach-part II: swarm tracking," in Proceedings of the American Control Conference (ACC '10), pp. 4750-4755, July 2010. 
[15] W. Ren, R. W. Beard, and E. M. Atkins, "Information consensus in multivehicle cooperative control," IEEE Control Systems Magazine, vol. 27, no. 2, pp. 71-82, 2007.

[16] Y. Hong, J. Hu, and L. Gao, "Tracking control for multiagent consensus with an active leader and variable topology," Automatica, vol. 42, no. 7, pp. 1177-1182, 2006.

[17] Y. Hong, G. Chen, and L. Bushnell, "Distributed observers design for leader-following control of multi-agent networks," Automatica, vol. 44, no. 3, pp. 846-850, 2008.

[18] S. Khoo, L. Xie, S. Zhao, and Z. Man, "Multi-surface sliding control for fast finite-time leader-follower consensus with high order SISO uncertain nonlinear agents," International Journal of Robust and Nonlinear Control, vol. 24, no. 16, pp. 2388-2404, 2014.

[19] S. V. Emelyanov, "Control of first order delay systems by mean of anastatic controller and nonlinear correction," Automation and Remote Control, vol. 20, no. 8, pp. 983-991, 1959.

[20] V. I. Utkin, "Variable structure systems with sliding modes," IEEE Transactions on Automatic Control, vol. 22, no. 2, pp. 212222, 1977.

[21] L. Fang, T. Li, Z. Li, and R. Li, "Adaptive terminal sliding mode control for anti-synchronization of uncertain chaotic systems," Nonlinear Dynamics, vol. 74, no. 4, pp. 991-1002, 2013.

[22] J. Huang, L. Sun, Z. Han, and L. Liu, "Adaptive terminal sliding mode control for nonlinear differential inclusion systems with disturbance," Nonlinear Dynamics, vol. 72, no. 1-2, pp. 221-228, 2013.

[23] J. Yang, S. Li, J. Su, and X. Yu, "Continuous nonsingular terminal sliding mode control for systems with mismatched disturbances," Automatica, vol. 49, no. 7, pp. 2287-2291, 2013.

[24] J. A. Moreno and M. Osorio, "A Lyapunov approach to secondorder sliding mode controllers and observers," in Proceedings of the 47th IEEE Conference on Decision and Control (CDC '08), pp. 2856-2861, December 2008.

[25] G. Bartolini, A. Pisano, and E. Usai, "An improved second-order sliding-mode control scheme robust against the measurement noise," IEEE Transactions on Automatic Control, vol. 49, no. 10, pp. 1731-1737, 2004.

[26] U. B. Kamble, J. O. Chandle, V. S. Lahire, and R. D. Langde, "Second order twisting sliding mode control of multi-agent network with input disturbance," in Proceedings of the 4th International Conference on Computing, Communications and Networking Technologies (ICCCNT '13), pp. 1-6, July 2013.

[27] S. Khoo, L. Xie, Z. Yu, and Z. Man, "Finite-time consensus algorithm of multi-agent networks," in Proceedings of the 10th International Conference on Control, Automation, Robotics and Vision (ICARCV'08), pp. 916-920, Hanoi, Vietnam, December 2008.

[28] S. Khoo, L. Xie, and Z. Man, "Robust finite-time consensus tracking algorithm for multirobot systems," IEEE/ASME Transactions on Mechatronics, vol. 14, no. 2, pp. 219-228, 2009.

[29] X. Huang, W. Lin, and B. Yang, "Global finite-time stabilization of a class of uncertain nonlinear systems," Automatica, vol. 41, no. 5, pp. 881-888, 2005.

[30] H. Du, S. Li, and P. Shi, "Robust consensus algorithm for second-order multi-agent systems with external disturbances," International Journal of Control, vol. 85, no. 12, pp. 1913-1928, 2012.

[31] R. Szupiluk, P. Wojewnik, and T. Zabkowski, "Smooth component analysis and MSE decomposition for ensemble methods in multi-agent environment," International Journal of Innovative
Computing, Information and Control, vol. 10, no. 4, pp. 14351445, 2014.

[32] A. Sedziwy, "Effective graph representation supporting multiagent distributed computing," International Journal of Innovative Computing, Information and Control, vol. 10, no. 1, pp. 101113, 2014.

[33] Q. Shen, B. Jiang, P. Shi, and J. Zhao, "Cooperative adaptive fuzzy tracking control for networked unknown nonlinear multi-agent systems with time-varying actuator faults," IEEE Transactions on Fuzzy Systems, vol. 22, no. 3, pp. 494-504, 2014.

[34] A. Levant, "Sliding order and sliding accuracy in sliding mode control," International Journal of Control, vol. 58, no. 6, pp. 12471263, 1993. 


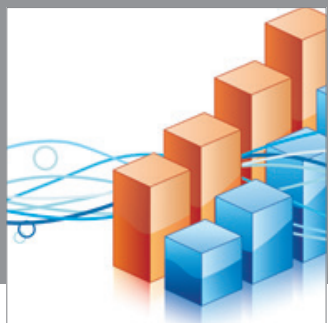

Advances in

Operations Research

mansans

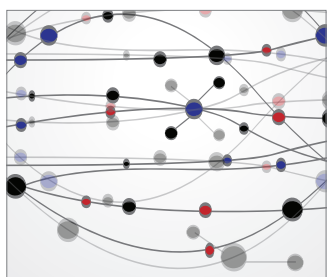

The Scientific World Journal
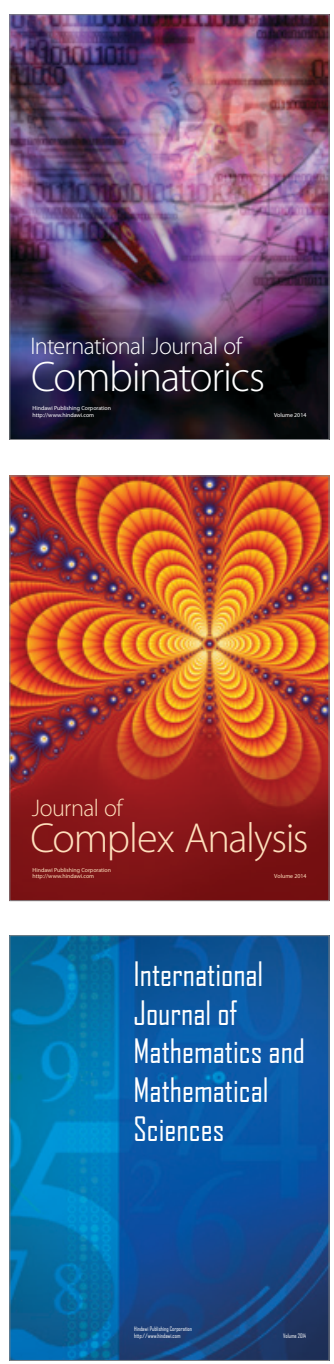
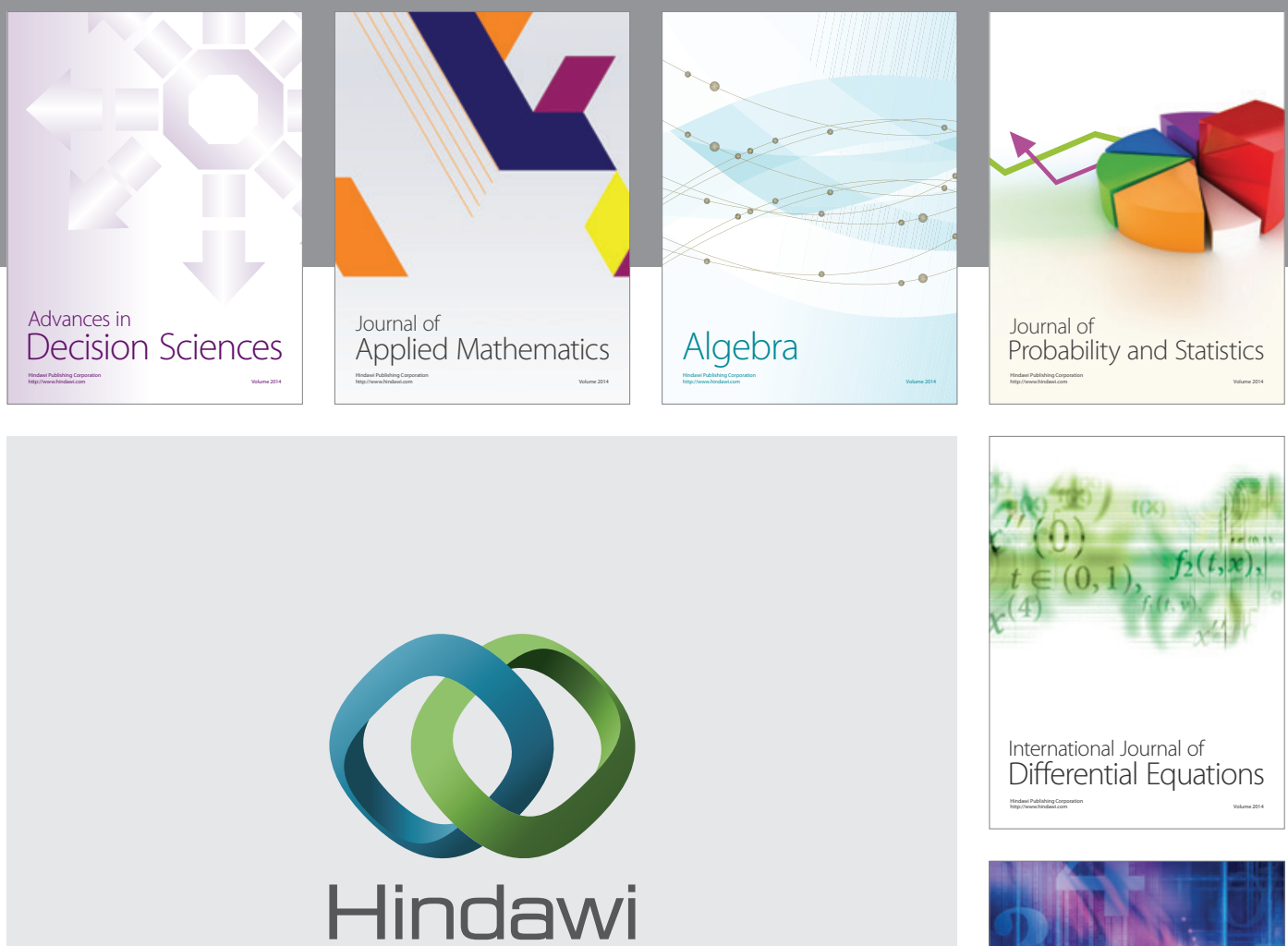

Submit your manuscripts at http://www.hindawi.com
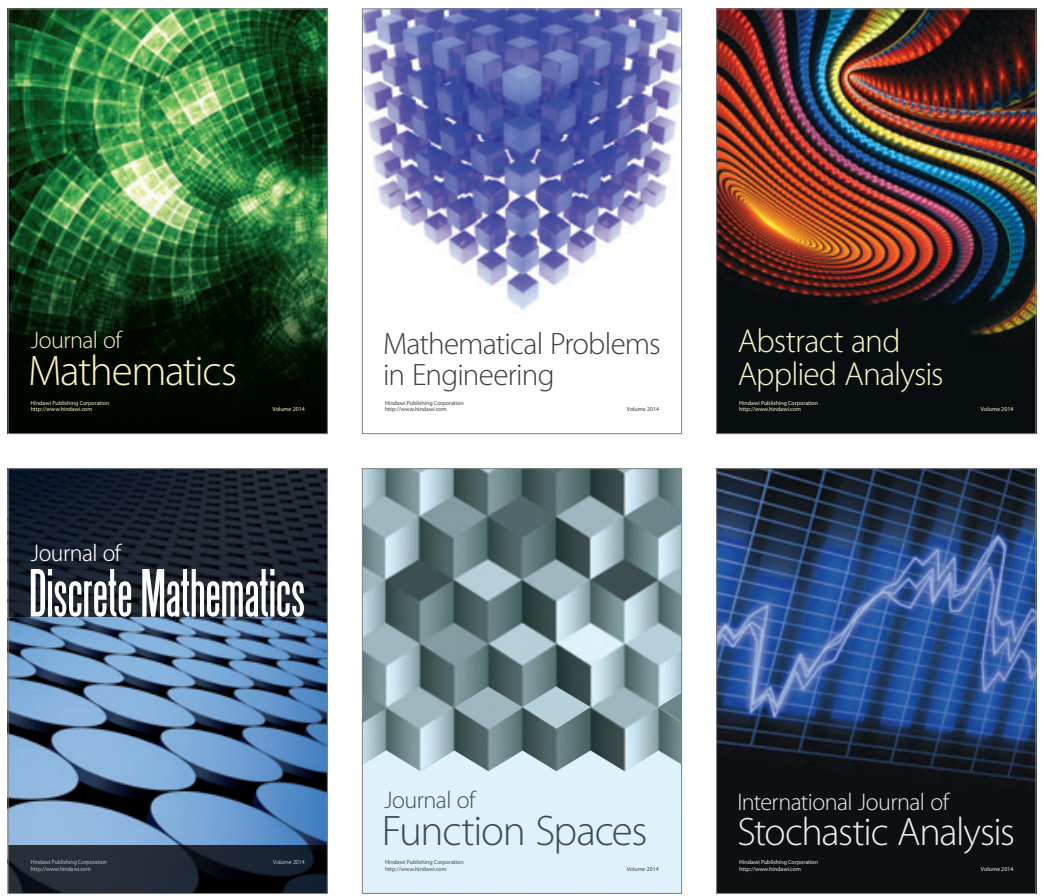

Journal of

Function Spaces

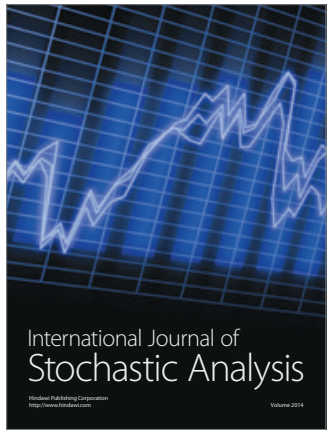

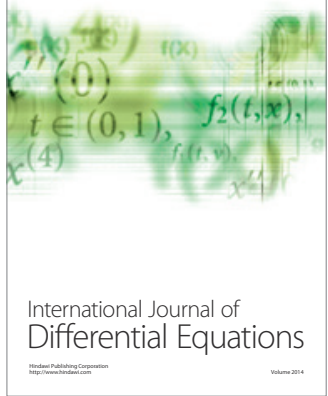
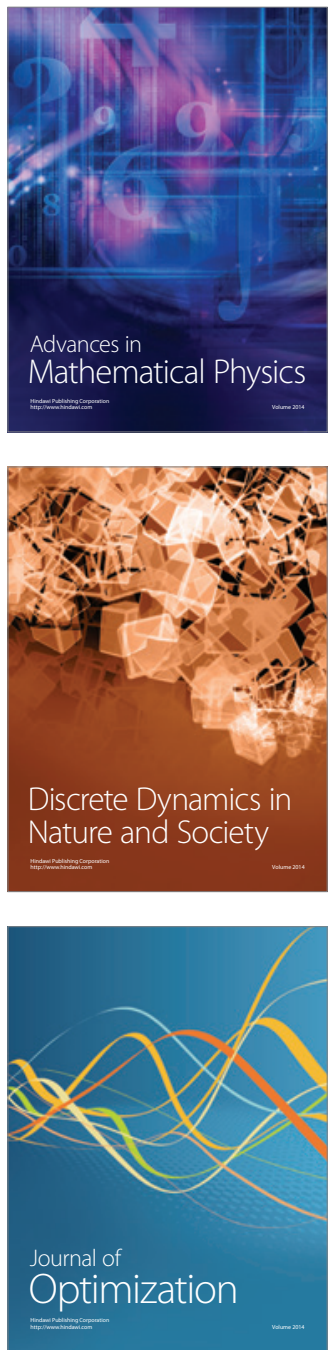